\title{
Approximate Solution of the Schrödinger Equation with Rosen-Morse Potential Including the Centrifugal Term
}

\author{
Akpan N. Ikot \\ Department of Physics, University of Uyo, Nigeria \\ E-mail: ndemikot2005@yahoo.com \\ Louis E. Akpabio \\ Department of Physics, University of Uyo, Nigeria \\ E-mail: Leabio2002@yahoo.com
}

\begin{abstract}
We derive approximate analytical solutions of the Schrödinger equation with Rosen-Morse potential via the Nikiforov-Uvarov method. The bound state energy eigenvalues are given in a closed form and the corresponding eigenfunctions are obtained in terms of the generalize Jacobi Polynomials and hypergeometrical function.
\end{abstract}

Keywords: Nikiforov-Uvarov methods, Schrödinger equation, Rosen-Morse potential

\section{Introduction}

The exact analytical solution of the Schrödinger equations are only possible with the angular momentum $l=0$ for some potential models. However, when $l \neq 0$, the Schrödinger equation can only be solve approximately for different suitable approximation scheme. One of such approximations are the convectional approximation scheme suggested by Greene and Aldrich and the new improved approximation scheme.

Many authors have investigated the bound state solution of Schrödinger approximately with exponential-line potential such as Eckart Potential, Hulthen potential and Manning Rosen Potential.

Presently, an alternative method for solving Schrödinger equation has been proposed. The method called Nikiforov-Uvarov (NU) is based on solving the second order linear differential equations by reducing to a generalize equation of hypergeometric type. This method had been used to solve the Schrödinger, Klein-Gordon, Dirac and Duffin-Kemner Petiau equations with exponential-like potentials such as Woods-Saxon, Huthen and Poschl-Teller, and generalize Posch-Teller.

In this paper, we attempt to solve approximately the Schrödinger equation with Rosen-Morse potential including the centrifugal term using the NU-Method. This potential plays a vital role in atomic, molecular and chemical physics since it can be used to describe molecular vibrations and to obtain the energy spectra of linear and nonlinear systems. The Rosen-Morse potential is one of the candidate potential used in describing the eigen function and energy spectra of quark confinement in quantum chromodynamics(QCD). The QCD prescribes the baryons to be constituted of three quarks in a colour singlet state. Bijker e tal exploits for the description of baryonic systems algebraic methods developed for the purposes of triatomic molecules. However an interesting dynamics limit of the three quark system is the one where two of the quarks are squeezed to one independent entity, a di-quark (QQ), while the third quark (Q) remains spectator. In that limit which corresponds to $U$ $(7) \rightarrow U(3) \times U(4)$, one can exploit the chain of reducing $U(4)$ down to $O(2)$. As a principle challenge still remaining unanswered is finding a suitable quark potential that explain the above scenario. The Rosen-morse potential with trigonometric function was use in Ref. [C. S. Jia, 2003] to explain the QCD quark-gluon dynamics. In their report they show that the shape of the potential capture the essentials of QCD quark-gluon dynamics where one gluon exchange gives rise to an effective coulomb-like potentials while the self-gluon interactions produce a linear confinement potential as established by lattice calculation of hadrons properties. It was also observed that the infinite wall shape of the trigonometric Rosen-Morse potential provides the regime suited for trapped but asymptotically free quarks. With this behaviour of this potential, one is tempted to conclude that this potential may be a good candidate for a quark potential of QCD traits. 
The organization of the paper is as follows. In section two we review the Nikiforov-Uvarov method. We find the bound solution of the Schrödinger with Rosen-Morse potential in section three. A brief conclusion is presented in section four.

\section{Review of Nikiforov-Uvarov Method}

The NU method is based on the solution of a generalized second order differential linear equation with special orthogonal functions. Therefore, a non-relativistic Schrödinger equation can be solved exactly using this method. For any given real or complex potential, the Schrödinger equation reduces to a generalized equation of hypergeometric type with an appropriate, $\mathrm{s}=\mathrm{s}(\mathrm{x})$ co-ordinate transformation. Thus, it can be written as follows:

$$
\psi^{\prime \prime}(s)+\frac{\bar{\tau}(s)}{\sigma(s)} \psi^{\prime}(s)+\frac{\bar{\sigma}(s)}{\sigma^{2}(s)} \psi(s)=0,
$$

where $\sigma(\mathrm{s})$ and $\bar{\sigma}(s)$ are polynomials at most second degree and $\bar{\tau}(s)$ is a first degree polynomials.

In order to find the exact solution to equation (1), we set the wave function as

$$
\psi(s)=\varphi(s) \chi(s),
$$

and on substituting equation (2) into equation (1) reduces equation (1) into hypergeometric type,

$$
\sigma(s) \chi^{\prime \prime}(s)+\tau(s) \chi^{\prime}(s)+\lambda \chi(s)=0,
$$

where the wave function $\varphi(\mathrm{s})$ is defined as the logarithmic derivative [G. Szego, 1939]

$$
\frac{\varphi^{\prime}(s)}{\varphi(s)}=\frac{\pi(s)}{\sigma(s)},
$$

where $\pi(s)$ is at most first degree polynomials.

Also, the hypergeometric type function $\chi(s)$ in equation (3) for a fixed $\mathrm{n}$ is given by the Rodriques relation

$$
\chi_{n}(s)=\frac{B_{n}}{\rho(s)} \frac{d^{n}}{d s^{n}}\left[\sigma^{\prime \prime}(s) \rho(s)\right]
$$

Where $B_{n}$ is the normalization constant and the weight function $\rho(s)$ must satisfy the condition [N. Cottas, 2004]

$$
\frac{d}{d s}(\sigma(s) \rho(s))=\tau(s) \rho(s)
$$

with

$$
\tau(s)=\bar{\tau}(s)+2 \pi(s) .
$$

To accomplish the conditions imposed on the weight function $\rho(s)$, it is necessary that the classical orthogonal polynomials $\tau(\mathrm{s})$ be equal to zero to some point of an interval $(\mathrm{a}, \mathrm{b})$ and its derivative at this interval at $\sigma(\mathrm{s})>0$ will be negative, that is

$$
\frac{d \tau(s)}{d s}<0 .
$$

Therefore, the function $\pi(s)$ and the parameter $\lambda$ required for the NU-method are defined as follows:

$$
\begin{gathered}
\pi(s)=\frac{\sigma^{1}-\bar{\tau}}{2} \pm \sqrt{\left(\frac{\sigma^{\prime}-\bar{\tau}}{2}\right)^{2}-\bar{\sigma}+k \sigma} \\
\lambda=k+\pi^{\prime}(s) .
\end{gathered}
$$

The k-values in the square-root of equation (9) is possible to evaluate if the expression under the square root must be square of polynomials. This is possible if its discriminate is zero. Thus, the new eigen-value equation for the Schrödinger equation becomes,

$$
\lambda=\lambda_{n}=-\frac{n d \tau}{d s}-\frac{n(n-1)}{2} \frac{d^{2} \sigma}{d s^{2}}, n=0,1,2, \ldots
$$


where $\tau(\mathrm{s})$ is as defined in equation (7) and on comparing equations (10) and (11), we obtain the energy eigen values.

\section{Solutions of the Schrödinger Equation with the Rosen-Morse Potential}

The generalized Rosen-Morse potential is given by

$$
v(x)=-v_{1} \operatorname{sech}_{1}^{2}(\alpha r)+v_{2} \tan (\alpha r),
$$

displayed in fig.1.The shape of this potential also captures the essentials behaviour of the QCD quark-gluon dynamics. The hyperbolic functions are defined as

$$
\begin{gathered}
\sinh \alpha r=\frac{e^{\alpha r}-e^{-\alpha r}}{2}, \quad \cosh \alpha r=\frac{e^{\alpha r}+e^{\alpha r}}{2} \\
\tanh \alpha r=\frac{\sinh \alpha r}{\cosh \alpha r} .
\end{gathered}
$$

The radial Schrödinger equation with Rosen-Morse potential is

$$
\begin{gathered}
-\frac{\hbar^{2}}{2 \mu}\left[\frac{d^{2}}{d r^{2}}+\frac{2}{r} \frac{d}{d r}-\frac{l(l+1)}{\hbar^{2} r^{2}}\right] \psi_{n l}(r) \\
+\left(v_{1} \sec h^{2}(\alpha r)+v_{2} \tanh \right) \psi_{n l}(r)=E_{n l} \psi_{n l}(r),
\end{gathered}
$$

where $l$ is the angular momentum number, $\mu$ is the reduced mass and $\frac{l}{r^{2}}$ is the centrifugal term.

Now introducing a new variable for the wave function as

$$
\psi_{n l}(r)=\frac{R(r)}{r}
$$

reduces equation (14) into

$$
\frac{d^{2} R(r)}{d r^{2}}+\frac{2 \mu}{\hbar^{2}}\left[E-v_{1} \sec h^{2}(\alpha r)-v_{2} \tanh (\alpha r)-\frac{\hbar^{2} l(l+1)}{2 \mu r^{2}}\right] R(r)=0
$$

Due to the centrifugal term in equation (16), this equation cannot be solved analytically when the angular momentum quantum number is not equal to zero.

Fortunately, approximate analytical solutions of with $l \neq 0$ has been devised to deal with centrifugal term. When $\alpha r \leq 1$, we use a new improved approximation scheme for the centrifugal term as [Y, Xu, 2010; C. S. Jia, 2009]

$$
\frac{1}{r^{2}}=4 \alpha^{2}\left[c_{0}+\frac{e^{-2 \alpha r}}{\left(1-e^{-2 \alpha r}\right)^{2}}\right]=4 \alpha^{2}\left[c_{0}+\frac{1}{4 \sinh ^{2} \alpha r}\right],
$$

where the parameter $c_{0}=\frac{1}{12}$ is a dimensional constant.

The convectional approximate scheme is obtain by setting $\mathrm{c}_{0}=0$ in equation (17). In order to apply the NU-method, we substitute equation (17) into equation (16) and using a new variable of the form.

$$
s=\tanh (\alpha r)
$$

we obtain the following, 


$$
\frac{d^{2} \varphi}{d s^{2}}-\frac{2 s}{\left(1-s^{2}\right)^{2}}\left[-\left(\beta^{2}+\pi^{2}\right) s^{2}+r^{2} s+\left(\varepsilon^{2}+\beta^{2}+\delta^{2}+\Lambda^{2}\right)\right]=0
$$

where

$$
\begin{gathered}
\varepsilon^{2}=-\frac{2 \mu E}{\hbar^{2} \alpha^{2}}, E>0, \beta^{2}=\frac{2 \mu v_{1}}{\hbar^{2} \alpha^{2}}, \\
\gamma^{2}=\frac{2 \mu v_{2}}{\hbar^{2} \alpha^{2}}, \quad \delta^{2}=4 l(l+1) c_{0}, \Lambda^{2}=l(l+1)
\end{gathered}
$$

are dimensionless parameters.

On comparing equation (19) with equation (1), we get

$$
\begin{gathered}
\bar{\tau}(s)=-2 s, \sigma(s)=1-s^{2}, \\
\bar{\sigma}(s)=-\left(\beta^{2}+\Lambda^{2}\right) s^{2}+\gamma^{2} s+\left(\varepsilon^{2}+\beta^{2}+\delta^{2}+\Lambda^{2}\right) .
\end{gathered}
$$

Substituting equations (20) into equation (9), we get

$$
\pi(s)= \pm \sqrt{-\left(\beta^{2}+\Lambda^{2}+k\right) s^{2}+\gamma^{2} s+\left(\varepsilon^{2}+\beta^{2}+\delta^{2}+\Lambda^{2}+k\right)}
$$

The expression in the square root must be square of polynomials in respect of the NU-method. Therefore, we can determine the constant $\mathrm{k}$ by the condition that the discriminate of the square root be zero, that is

$$
K_{ \pm}=-\frac{\left(2 \beta^{2}+2 \wedge^{2}+\varepsilon^{2}+\delta^{2}\right) \pm \sqrt{\left(\varepsilon^{2}+\delta^{2}\right)^{2}-\gamma^{4}}}{2},
$$

In view of equation (22), we find new possible functions of $\pi(s)$ for each $\mathrm{k}$ as

$$
\pi(s) z^{ \pm} \frac{1}{2}\left\{\begin{array}{c}
\left(\sqrt{\varepsilon^{2}+\delta^{2}+\gamma^{2}}\right) s+\sqrt{\varepsilon^{2}+\delta^{2}+\gamma^{2}} \\
\text { for, } \quad k=-\frac{\left(2 \beta^{2}+2 \Lambda^{2}+\varepsilon^{2}+\delta^{2}\right)+\sqrt{\left(\varepsilon^{2}+\delta^{2}\right)-\gamma^{4}}}{2} \\
\left(\sqrt{\varepsilon^{2}+\delta^{2}-\gamma^{2}}\right) s-\sqrt{\varepsilon^{2}+\delta^{2}+\gamma^{2}}, \text { for } \\
\text { for, } \quad k=-\frac{\left(2 \beta^{2}+2 \Lambda^{2}+\varepsilon^{2}+\delta^{2}\right)-\sqrt{\left(\varepsilon^{2}+\delta^{2}\right)^{2}-\gamma^{4}}}{2}
\end{array}\right.
$$

For the polynomial of $\tau=\tau+2 \pi(s)$, which has a negative derivative, we get

$$
\begin{aligned}
& k=-\frac{\left(2 \beta^{2}+2 \Lambda+\varepsilon^{2}+\delta^{2}\right)-\sqrt{\left(\varepsilon^{2}+\delta^{2}\right)^{2}-\gamma^{4}}}{2} \\
& \pi(s)=-\frac{1}{2}\left(\sqrt{\varepsilon^{2}+\delta^{2}-\gamma^{2}}\right) s+\frac{1}{2} \sqrt{\varepsilon^{2}+\delta^{2}-\gamma^{2}}
\end{aligned}
$$

Now using $\lambda=k+\pi^{\prime}(s)$ together with the values $\mathrm{k}$ and $\pi(s), \bar{\tau}$, we obtain

$$
\begin{gathered}
\tau(s)=-2 s-\frac{1}{2}\left(\sqrt{\varepsilon^{2}+\delta^{2}-\gamma^{2}}\right) s+\frac{1}{2} \sqrt{\varepsilon^{2}+\delta^{2}-\gamma^{2}} \\
\lambda=-\frac{\left(2 \beta^{2}+2 \Lambda^{2}+\varepsilon^{2}+\delta^{2}\right)-\sqrt{\left(\varepsilon^{2}+\delta^{2}\right)^{2}-\gamma^{4}}}{2}-\frac{1}{2} \sqrt{\varepsilon^{2}+\delta^{2}-\gamma^{2}}
\end{gathered}
$$


Also from the definition of $\lambda_{n}$, we find

$$
\lambda_{n}=-2 n+n / 2 \sqrt{\varepsilon^{2}+\delta^{2}-\gamma^{2}}+n(n-1) .
$$

Comparing equations (27) and (28), we obtain approximately the energy spectrum of the radial Schrödinger equation with Rosen-Morse potential as

$$
\begin{gathered}
-\sqrt{\left(\varepsilon^{2}+\delta^{2}\right)-v^{4}}-\sqrt{\left(\varepsilon^{2}+\delta^{2}-\gamma^{2}\right)}-n \sqrt{\left(\varepsilon^{2}+\delta^{2}-\gamma^{2}\right)} \\
-\left(\varepsilon^{2}+\delta^{2}\right)=2 \beta^{2}+2 \Lambda^{2}+2 n(n-1)-4 n
\end{gathered}
$$

or simplifying equation (29) yields

$$
\begin{array}{r}
\left(\varepsilon^{2}+\delta^{2}\right)^{2}-\left\{2\left[2 \beta^{2}+2 \Lambda^{2}+2 n(n-1)-4 n\right]\right. \\
+3(n+1)]\}\left(\varepsilon^{2}+\delta^{2}\right)+\left(\gamma^{2}+3(n+1) \gamma^{2}\right) \\
+\left[2 \beta^{2}+2 \Lambda^{2}+2 n(n-1)-4 n\right]^{2}
\end{array}
$$

Solving equation (30) explicitly, we obtain the energy spectrum as

$$
\begin{gathered}
E=-\frac{\hbar^{2} \alpha^{2}}{2 \mu}\left\{\left[\frac{4 \mu v_{1}}{\hbar^{2} \alpha^{2}}+2 \ell(\ell+1)\left[1-2 c_{0}\right]\right.\right. \\
+2 n(n-1)+3 / 2(n+1)+1 / 2-4 n] \\
\pm\left(\frac{4 \mu v_{1}}{\hbar^{2} \alpha^{2}}+2 \ell[\ell+1]+2 n(n-1)-4 n+3 / 2(n+1)+1 / 2\right. \\
\left.\left.+\frac{2 \mu v_{2}}{\hbar^{2} \alpha^{2}}[1+3(n+1)]-\left[\left(\frac{4 \mu v_{1}}{\hbar^{2} \alpha^{2}}\right)+2 \ell(\ell+1)+2(n-1)-4 n\right]^{2}\right)^{\frac{1}{2}}\right\}
\end{gathered}
$$

where we have use equation (19b) in obtaining (31) explicitly.

From equation (6), we obtain the weight function $\rho(s)$ as

$$
\rho(s)=(1-s) \frac{\left(1+\sqrt{\varepsilon^{2}+\delta^{2}-\gamma^{2}}\right)}{4}(1-s)^{\left(1-\sqrt{\varepsilon^{2}+\delta^{2}-\gamma^{2}}\right) 1 / 4},
$$

and on substituting equation (32) into the Rodriques relation of equation (5), we get,

$$
\begin{gathered}
\chi(s)=B_{n}(1-s)^{-1 / 4\left(1+\sqrt{\varepsilon^{2}+\delta^{2}-r}\right)}(1+s)^{1 / 4\left(1-\sqrt{\varepsilon^{2}+\delta^{2}-r}\right)} \\
x \frac{d^{n}}{d s^{n}}\left[(1-s)^{2}(1-s)^{-1 / 4\left(1+\sqrt{\varepsilon^{2}+\delta^{2}-r}\right)}(1+s)^{1 / 4\left(1-\sqrt{\varepsilon^{2}+\delta^{2}-r}\right)}\right] \\
=B_{n} P_{n}^{(\mu+v), \mu-v}(s),
\end{gathered}
$$


where $\mu=1 / 4, v=1 / 4^{\sqrt{\varepsilon^{2}+\delta^{2}-r^{2}}}$ and $\mathrm{P}_{\mathrm{n}}$ is the Jacobi polynomials.

The remaining part of the wave function in equation (4) is found as

$$
\varphi(s)=(1-s)^{2(\mu+v)}(1+s)^{2(\mu-v)}
$$

Combining the Jacob polynomials of equation (33) and equation (34), we find the s-wave function as

$$
R_{n}(s)=C_{n}(1-s)^{2^{(\mu+v)}}(1+s)^{2^{(\mu+v)}} P_{n}^{(\mu+v), \mu-v}(s)
$$

where $\mathrm{C}_{\mathrm{n}}$ is the new normalization constant and it is determined by the condition $\int_{-\infty}^{\infty} R_{n}(s) d s=1$.

\section{Conclusion}

In this research work, we investigated the bound state solutions of the Schrödinger equation with Rosen-Morse potential for any arbitrary angular momentum number $l$ approximately. With the new improved scheme for the centrifugal term, we obtained the energy eigenvalues and the unnormalized wave function in terms of the hypergeometric functions via the Nikiforov-Uvarov method. The shape of this potential captures the essential traits of the quark-gluon dynamics of QCD and it is a good choice that can be employed in the calculations of spectroscopic properties of non-strange resonances.

Finally, our results are in consistent with those of refs. [C. S. Jia, 2003; 2002].

\section{Acknowledgement}

We acknowledge the financial support of Nandy-Leabio grants No. NL5563.

\section{References}

A. Berkedemir, C. Berkdemir and R. Sever. Quant-Ph/0410153.

A. F. Nikiforov, and V. B. Uvaroo. (1988). Special Function of Mathematical Physics, Birkhausev, Basel.

C. B. Compean and M. Kirchbach. (2006). Proceedings of the Mini-Workshop-Progress in Quark Models, Angular Momentum Dependent Quark Potential of QCD Traits and Dynamical O(4) Symmetry, (Bled, Slovenia).

C. Berkdemir, A. Berkdemir and R. Sever. (2004). Arxir: nucl-ph/0412021.

C. Berkedemir, A-Berkdemir and R. Saver. (2005). Phys. Rev., C 72, 027001.

C. S. Jia, Y. Li, Y. Sun, J. Y. Liu, L. T. sun. (2003). Phys. Lett. A 311, 115.

C. S. Jia, T. Chen and L. G. Cui. (2009). Phys. Lett. A 373, 1621.

C. S. Jia, X. L. Zeng, L. T. Sun. (2002). Phys. Lett., A 294. 185.

F. Taskin. (2009). Int. J. Theor. Phys., 48, 1142.

G. Szego. (1939). Orthogonal Polynomials, (American Mathematical Society, New York.

H. Egrifes, D. Demirhan and F. Buyukkihc. (1999). Physics Scripta, 59, 90.

H. Egrites, D. Dimerhan and F. Buyukkihc. (1999). Physics scripta, 195.

L. Chetouchi, L. Guechi, A. Lecheheb, T. F. Hamman and A. Messouber. (1996). Physic A, 234, 529.

L. H. Zhang, X. P. Li and C. S. Yia. (2008). Phys. Lett., A 372, 2201.

N. Cottas. (2004). CEJP, 2, 456.

N. Rosen and P. M. Morse. (1932). Phys. Rev., 42, 192.

O. Yesiltas, M. Simsek, R. Sever and C. Tezcan. (2003). Phys. Scripta, T67, 472.

R. Biyker, F. Iachello, A. Leviatan, Yu:F Smirnov. (2001). Phys. Rev. D44, 114005.

R. L. Greene and C. Aldrich. (1976). Phys. Rev., A. 14, 2363.

S. Flugge. (1974). Practical Quantum Mechanics, (Springer, - Verlag, Berlin).

V. H. Badalov, H. I. Almadov and A. I Almadov. (2009). Arxio: Math-ph/0905.273. 
W. C. Qiang and S. H. Dong. (2008). Phys. Lett., A 372, 4789.

Y, Xu, S. He and C. S. Jia. (2010). Phys. Scripta, 81, 045001.

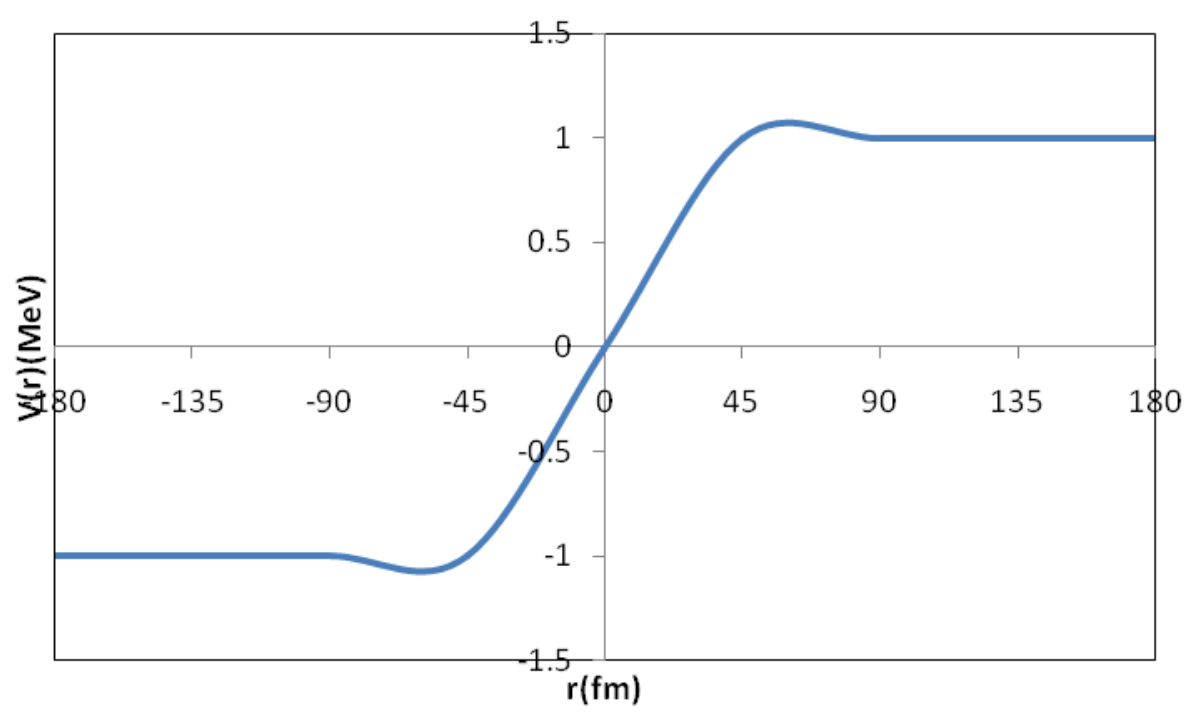

Figure 1. The variation of Rosen-Morse potential V(r) with radius $r$. The shape of the potential shows a typical QCD quark-gluon dynamics 